\begin{tabular}{|c|l|}
\hline Title & On global weak solutions of the nonstationary two-phase Stokes flow \\
\hline Author(s) & Giga, Y oshikazu; Takahashi, Shuji \\
\hline Citation & Hokkaido University Preprint Series in Mathematics, 149, 2-25 \\
\hline Issue Date & 1992-05 \\
\hline DOI & 10.14943/83293 \\
\hline Doc URL & http://hdl.handle.net/2115/68895 \\
\hline Type & bulletin (article) \\
\hline File Information & pre149.pdf \\
\hline
\end{tabular}

Instructions for use 
ON GLOBAL WEAK SOLUTIONS OF THE NONSTATIONARY TWO-PHASE STOKES FLOW

\author{
Y. Giga and S. Takahashi
}

Series $\sharp 149$. May 1992 


\section{HOKKAIDO UNIVERSITY \\ PREPRINT SERIES IN MATHEMATICS}

$\sharp 122$ : Y.-G. Chen, Blow-up solutions to a finite difference analogue of $u_{t}=\Delta u+u^{1+\alpha}$ in $N$-dimensional balls, 31 pages. 1991.

\#123: A. Arai, Fock-space representations of the relativistic supersymmetry algebra in the two-dimensional spacetime, 13 pages. 1991.

$\$ 124$ : S. Izumiya, The theory of Legendrian unfoldings and first order differential equations, 16 pages. 1991.

$\sharp 125$ : T. Hibi, Face number inequalities for matroid complexes and Cohen-Macaulay types of Stanley-Reisner rings of distributive lattices, 17 pages. 1991.

\#126: S. Izumiya, Completely integrable holonomic systems of first order differential equations, 35 pages. 1991.

\#127: G. Ishikawa, S. Izumiya and K. Watanabe, Vector fields near a generic submanifold, 9 pages. 1991.

\#128: A. Arai, I. Mitoma, Comparison and nuclearity of spaces of differential forms on topological vector spaces, 27 pages. 1991.

$\sharp 129$ : K. Kubota, Existence of a global solution to a semi-linear wave equation with initial data of non-compact support in low space dimensions, 53 pages. 1991.

$\sharp 130$ : S. Altschuler, S. Angerent and Y. Giga, Mean curvature flow through singularities for surfaces of rotation, 62 pages. 1991.

$\sharp 131$ : M. Giga, Y. Giga and H. Sohr, $L^{p}$ estimates for the Stokes system, 13 pages. 1991.

$\sharp 132$ : Y. Okabe, T. Ootsuka, Applications of the theory of $\mathrm{KM}_{2} \mathrm{O}$-Langevin equations to the non-linear prediction problem for the one-dimensional strictly stationary time series, 27 pages. 1992.

$\sharp 133$ : Y. Okabe, Applications of the theory of $\mathrm{KM}_{2} \mathrm{O}$-Langevin equations to the linear prediction problem for the multi-dimensional weakly stationary time series, 22 pages. 1992.

\# 134: P. Aviles, Y. Giga and N. Komuro, Duality formulas and variational integrals, 22 pages. 1992.

135: S. Izumiya, The Clairaut type equation, 6 pages. 1992.

\#136: S. Izumiya, Singular solutions of first order differential equations, 6 pages. 1992.

$\sharp$ 137: S. Izumiya, W.L. Marar, The Euler characteristic of a generic wave front in a 3-manifold, 6 pages. 1992.

$\sharp$ 138: S. Izumiya, W.L. Marar, The Euler characteristic of the image of a stable mapping from a closed $n$-manifold to a $(2 n-1)$-manifold, 5 pages. 1992.

$\sharp 139$ : Y. Giga, Z. Yoshida, A bound for the pressure integral in a plasma equilibrium, 20 pages. 1992.

$\sharp 140$ : S. Izumiya, What is the Clairaut equation ?, 13 pages. 1992.

$\sharp 141$ : H. Takamura, Weighted deformation theorem for normal currents, 27 pages. 1992.

$\sharp 142$ : T. Morimoto, Geometric structures on filtered manifolds, 104 pages. 1992.

\#143: G. Ishikawa, T. Ohmoto, Local invariants of singular surfaces in an almost complex four-manifold, 9 pages. 1992.

$\sharp 144$ : K. Kubota, K. Mochizuki, On small data scattering for 2-dimensional semilinear wave equations, 22 pages. 1992.

$\sharp 145$ : T. Nakazi, K. Takahashi, Hyponormal Toeplitz operators and extremal problems of Hardy spaces, 30 pages. 1992.

$\sharp 146$ : N. Hayashi, T. Ozawa, Remarks on nonlinear Schrödinger equations in one space dimension, 10 pages. 1992.

$\sharp 147$ : M. Sato, Interface evolution with Neumann boundary condition, 16 pages. 1992.

$\sharp 148$ : $\quad$ Y. Okabe, Langevin equations and causal analysis, 49 pages. 1992 . 


\title{
ON GLOBAL WEAK SOLUTIONS \\ OF THE NONSTATIONARY TWO-PHASE \\ STOKES FLOW
}

Yoshikazu Giga' and Shuji Takahashi

\begin{abstract}
A global-in-time weak solution of the nonstationary two-phase Stokes flow is constructed for arbitrary given initial domains (under periodic boundary condition) when two viscosities are close. Our solution tracks the evolution of the interface after it develops singularities. The theory of viscosity solutions is adapted to solve the interface equation. Surface tension effects are ignored here.
\end{abstract}

Key words. global solutions, two-phase Stokes system, interface equation, generalized evolution, upper semicontinuous convexification

AMS(MOS) subject classifications (1990). $\quad$ 35Q30, 35R35, 58C06, 76T05

1. Introduction. This paper studies the dynamics of the interface (free boundary) of two immiscible incompressible viscous fluids with same constant density, say one. We are interested in slow motions so that each fluid velocity satisfies the Stokes equations with different viscosities. The interface is assumed to move with the fluid velocities. No surface tension on the interface is considered in this paper.

Let $\nu_{ \pm}$be the viscosities of each fluid. Let $\Omega_{ \pm}(t)$ the disjoint open sets in a bounded open rectangle $R\left(\subset \mathbf{R}^{n}(n \geqslant 2)\right)$ occupied with the fluids of viscosities $\nu_{ \pm}$at time $t$, respectively. The complement of the union of $\Omega_{+}(t)$ and $\Omega_{-}(t)$ is called the interface and

\footnotetext{
${ }^{1}$ Department of Mathematics, Hokkaido University, Sapporo 060, Japan

${ }^{2}$ Department of Mathematical Science, Faculty of Science and Engineering, Tokyo Denki University, Hatoyama, Saitama 350-03, Japan
} 
denoted by $\Gamma(t)$. To write down the equation we assume that the interface $\Gamma(t)$ is a smooth hypersurface so that $\Gamma(t)$ is the boundary between $\Omega_{+}(t)$ and $\Omega_{-}(t)$. Let $u_{ \pm}=u_{ \pm}(t, x)$ and $\pi_{ \pm}=\pi_{ \pm}(t, x)$ denote the velocities and pressures of fluids with viscosities $\nu_{ \pm}$, respectively. The motion of the fluids determines the dynamics of the interface. Let $V=V(t, x)$ denote the speed of $\Gamma(t)$ at $x \in \Gamma(t)$ in the normal direction $n$ from $\Omega_{+}(t)$ to $\Omega_{-}(t)$. We consider an interface equation for $\Gamma(t)$ :

$$
V=u_{+} \cdot \mathbf{n} \text { on } \Gamma(t) \text { with initial data } \Omega_{ \pm}(0)=\Omega_{ \pm 0}
$$

coupled with the incompressible Stokes system:

$$
\begin{array}{ll}
\partial_{t} u_{ \pm}-\nu_{ \pm} \Delta u_{ \pm}+\nabla \pi_{ \pm}=\nabla \cdot f_{ \pm}, & \text {in }(0, T) \times \Omega_{ \pm}(t), \\
\nabla \cdot u_{ \pm}=0, & \text { in }(0, T) \times \Omega_{ \pm}(t), \\
u_{+}=u_{-}, & \text {on } \Gamma(t), \\
T_{+}\left(u_{+}, \pi_{+}\right) \cdot \mathrm{n}=T_{-}\left(u_{-}, \pi_{-}\right) \cdot \mathrm{n}, & \text { on } \Gamma(t), \\
u_{ \pm}(0, x)=0, & \text { in } \Omega_{ \pm}(0),
\end{array}
$$

where $T_{ \pm}\left(u_{ \pm}, \pi_{ \pm}\right):=\nu_{ \pm} D\left(u_{ \pm}\right)-\pi_{ \pm} I$ denotes the stress tensors with

$$
D(u)=\left(D_{k \iota}(u)\right):=\frac{\partial u^{k}}{\partial x_{\ell}}+\frac{\partial u^{\ell}}{\partial x_{k}} .
$$

Here $0<\nu_{-}<\nu_{+}<\infty, 0<T \leqslant \infty$ and $f=\left(f_{i j}(t, x)\right)(i, j=1, \cdots, n)$. The initial velocities are assumed to be zero for simplicity.

Our goal is to construct global weak solutions of the two-phase Stokes system (1.1)(1.6) for arbitary given initial domains $\Omega_{ \pm 0}$ and external forces $f_{ \pm}$under the assumption that $\nu_{+}$and $\nu_{-}$are close. Here we impose periodic boundary conditions to avoid technical difficulties. Although it is possible to construct local solutions (cf. [De2]), there is an intrinsic difficulty to construct global solutions since the interface $\Gamma(t)$ may develop singularities in a finite time.

We first introduce a weak formulation of the transport equation (1.1). Since the boundary of our domain $\Omega_{ \pm}(t)$ may not be regular, we consider a generalized evolution of (1.1) through a level set of an auxiliary function. This idea goes back to [ESou]. Recently, 
the level set approach is extended to other equations including the mean curvature flow equations (cf. [ES], [CGG1]). However, our velocity field $u$ is merely continuous, so one cannot apply these known theories directly to our setting. We are forced to extend the usual definition of generalized evolutions to (1.1)(cf. [ESou]). It turns out that our generalized evolution uniquely exists for any initial domains and any continuous velocity $u$.

Using generalized evolutions of (1.1), we next introduce a step function $\nu$ to give an weak formulation of (1.2)-(1.6). The region occupied with high (resp. low) viscous fluid corresponds to the place where $\nu$ takes the value $\nu_{+}$(resp. $\left.\nu_{-}\right)$. The interface corresponds to a jump discontinuity of $\nu$. The velocity $u$ is defined by $u=u_{+}$on $\Omega_{+}$and $u=u_{-}$on $\Omega_{-}$, and also the pressure $\pi$ is defined in the same manner. The system (1.2) and (1.5) is formally equivalent to

$$
u_{t}-\nabla \cdot(\nu D(u))+\nabla \pi=\nabla \cdot f, \quad \text { in }(0, T) \times \mathbf{T}
$$

where $T$ is the torus obtained by identifying each ends of $R$. The condition (1.5) is implicit in (1.7). The condition (1.4) is automatic if $u$ is assumed to be continuous. We thus obtain an weak formulation of (1.1)-(1.6).

To construct a solution we seek a fixed point of the mapping defined as follows. For a continuous function $v$ we solve (1.1) with $u_{+}=v$ and find generalized evolutions $\Omega_{ \pm}^{v}$. Let $\nu=\nu_{v}$ be a step function with $\nu=\nu_{ \pm}$on $\Omega_{ \pm}^{v}$ and $\nu=\left(\nu_{+}+\nu_{-}\right) / 2$ outside $\Omega_{ \pm}^{v}$. We next solve (1.7) with $\nabla \cdot u=0$ and $u(0, x)=0$, and obtain a mapping $S: v \longmapsto u$. Unfortunately $S$ is not continuous, so Leray-Schauder's fixed point theory does not apply. We extend mapping $S$ to an upper semi-continuous convex set-valued mapping so that we apply Kakutani's fixed point theory. To apply Kakutani's theory we need a compactness which follows from a priori $L^{p}$ estimates (for large $p$ ) for the Stokes system (1.7) and $\nabla \cdot u=0$ with discontinuous viscosity. A perturbation argument similar to [Cam] and [GY] is applied here. To get the $L^{p}$ estimates for large $p$ we need to assume that $\left(\nu_{+}-\nu_{-}\right) / \nu_{+}$ is sufficiently small.

In [GGI] and [GY] global solutions for the interface equations coupled with other equations are studied in different contexts.

There are related free boundary problems for one-phase incompressible viscous fluid motion. Solonnikov extensively studied the evolution of the free boundary when the initial 
surface is a connected boundary of a bounded domain. He constructed a unique local smooth solution for $\sigma=0$ in [Sol 5] and $\sigma>0$ in [Sol 6], where $\sigma$ is the surface tension. If the data is close to some equilibrium state, he showed that his solution can be extended globally in time; see [Sol $1,2,3]$ for $\sigma>0$ and [Sol 4] for $\sigma=0$.

The same problem is studied when the domain is occupied with fluid like an ocean with finite depth whose top is the free boundary. Local existence is established by Beale [Be 2] and Allain [Al] for $\sigma>0$ and by Beale [Be1] for $\sigma=0$. Global-in-time existence of smooth solution is established by Beale [Be 2] for $\sigma>0$ and Sylvester [Sy] for $\sigma=0$. Note that the case $\sigma=0$ is more difficult for establishing global existence because $\sigma>0$ gives some regularizing effect.

For the two-phase Navier-Stokes system, using a priori estimates in [De1], Denisova [De2] constructed a local solution with or without the surface tension. Tanaka [Tana] proved a global existence for $\sigma>0$ when the initial surface is close to some equilibrium state.

Our problem (1.1)-(1.6) is regarded as the two-phase Stokes system with no surface tension. The only difference between our problem and the two-phase Navier-Stokes system mentioned above is that our equations for the fluid motion are not the Navier-Stokes equations but the Stokes equations. So far even to our problem no global smooth solutions are constructed for nontrivial initial data.

Finally we point out that Kohn and Lipton [KL] discussed homogenization problem for the two-phase Navier-Stokes flow with no surface tension in a formal level.

We note that two-phase problem for compressible viscous fluid is extensively studied by Tani. We refer to [Tani $1,2,3]$.

This work was done while the second author was a graduate student in the Department of Mathematics, Hokkaido University. The work of the first author was partially supported by the Inamori Foundation.

We are grateful to Professor Hitoshi Ishii and Professor Hisashi Okamoto for criticism of solutions of the transport equations.

2. Interface equations. We consider the motion of interfaces with a given 
speed under periodic boundary conditions. For $\alpha_{i}>0(i=1, \cdots, n)$ let $R$ be a bounded rectangle in $R^{n}$ of the form

$$
R=\left\{\left(x_{1}, \cdots x_{n}\right) \in \mathbf{R}^{n} ; 0 \leqslant x_{i} \leqslant \alpha_{i}, 1 \leqslant i \leqslant n\right\}
$$

We identify faces $x_{i}=0$ and $x_{i}=\alpha_{i}(1 \leqslant i \leqslant n)$ of $R$ to get an $n$-dimensional flat torus T. A motion of interfaces in $R$ under periodic boundary conditions is interpreted as that in $\mathbf{T}$. We consider $\mathbf{T}$ rather than $\mathbf{R}^{\boldsymbol{n}}$ for technical convenience because $\mathbf{T}$ is compact and has no boundary. The periodic boundary condition is important because it is often used in numerical experiments.

Let $\Omega_{+}$and $\Omega_{-}$be disjoint open sets in $M=[0, \infty) \times T$. Let $\Gamma$ denote the complement of the union of $\Omega_{+}$and $\Omega_{-}$in $M$. Physically, $\Gamma(t)$ is called an interface at time $t$ bounding two phases $\Omega_{ \pm}(t)$ of fluids. Here $W(t)$ denotes the cross-section of $W \subset M$ at time $t$, i.e.,

$$
W(t)=\{\boldsymbol{z} \in \mathbf{T} ;(t, x) \in W\}
$$

Suppose that $\Gamma(t)$ is a smooth hypersurface and let $\mathbf{n}$ denote the unit normal vector field pointing from $\Omega_{+}(t)$ to $\Omega_{-}(t)$. Let $V=V(t, x)$ denote the speed of $\Gamma(t)$ at $x \in \Gamma(t)$ in the direction $\mathbf{n}$. Suppose that $u: \bar{Q} \rightarrow \mathbf{R}^{n}$ is a continuous vector field, i.e., $u \in C(\bar{Q})$ where $Q=(0, T) \times \mathbf{T}(0<T \leqslant \infty)$ and that $\bar{Q}$ denotes the closure of $Q$ in $M$. Here and hereafter we do not distinguish the space of real, vector or tensor valued functions. The equation for $\Gamma(t)$ we consider here is

$$
V=u \cdot \mathbf{n}, \quad \text { on } \Gamma(t)
$$

where - denotes the standard inner product in $\mathbf{R}^{n}$.

If $u(t, x)$ is Lipschitz continuous in $x$ (uniformly in $t$ ), one can construct a unique short time classical solution for a given smooth initial data $\Gamma(0)$ by a method of characteristics. In the periodic case a unique global-in-time weak solution is constructed in [GGI] by a level set approach developed by Y.-G. Chen, Giga and Goto [CGG1] and Evans and Spruck [ES]; see also [ESou]. However, if $u$ is merely continuous, classical solutions may not exist even for a short time and they are not uniquely determined by the initial data even if they exist. The level set approach in [GGI] does not apply to this case so we are forced to 
extend the approach. By the way in [CGG2] we actually need to assume a uniform bound on the gradient of $T$ in [CGG2, (1.6)] and of $\omega$ in [CGG2, (2.13)] although it is not written there.

Largest and smallest solutions. Let $u \in C(\bar{Q})$ and $a \in C(\mathrm{~T})$. We say $\psi: Q \longrightarrow \mathrm{R}$ is a subsolution of

$$
\begin{aligned}
& \psi_{t}+(u \cdot \nabla) \psi=0, \text { in } Q \\
& \psi(0, x)=a(x)
\end{aligned}
$$

if $\psi$ is a viscosity subsolution of $(2.2)$ on $Q$ and $\psi_{*}(0, x)=a(x)$, where $h_{*}$ denotes the lower semicontinuous envelope of $h: I \longrightarrow \mathbf{R}$, i.e.,

$$
h_{*}(y)=\lim _{\epsilon \downarrow 0} \inf \{h(z) ;|z-y|<\epsilon, z \in I\}, y \in \bar{I} \text {. }
$$

If $-\psi$ is a subsolution of (2.2)-(2.3) with $-\psi(0, x)=-a(x)$, we say $\psi$ is a supersolution of (2.2)-(2.3). If $\psi$ is both super- and subsolution of (2.2)-(2.3), we simply say $\psi$ is a solution of (2.2)-(2.3). For a general theory of viscosity solutions see [CIL].

As well known there is a comparison theorem on solutions provided that $|\nabla u|$ is uniformly bounded. However, for general $u \in C(\bar{Q})$ there is no uniqueness of solutions of (2.2)-(2.3). We thus consider largest and smallest solutions. Let $\lambda$ (resp. $\sigma$ ) be a solution of (2.2)-(2.3). We say $\lambda$ (resp. $\sigma$ ) is a largest (resp. smallest) solution if $\lambda \geqslant \psi$ (resp. $\sigma \leqslant \psi$ ) for all other solutions $\psi$ of (2.2)-(2.3).

Proposition 2.1. (i) Suppose that $\psi$ is a viscosity sub-(super)solution of (2.2) on $Q$, where $u \in C(\bar{Q})$. Then $\psi$ is also a viscosity sub-(super)solution of

$$
\begin{aligned}
\psi_{t}-L|\nabla \psi| & =0 \\
\text { resp. } \quad \psi_{t}+L|\nabla \psi| & =0)
\end{aligned}
$$

on $Q$ with $L=\sup _{Q}|u|$.

(ii) Suppose that $\psi$ is a viscosity super-(sub)solution of (2.4) (resp. (2.5)). Then $\psi$ is also a viscosity super-(sub)solution of (2.2) on $Q$. 
Proof. We only present the proof of (i) when $\psi$ is a viscosity subsolution of (2.2) because the remaining three cases can be proved similarly. Suppose that $\zeta \in C^{2}(Q)$ and $\left(t_{0}, x_{0}\right) \in Q$ satisfy

$$
\max _{Q}(\psi-\zeta)=(\psi-\zeta)\left(t_{0}, x_{0}\right)
$$

Since $\psi$ is a viscosity subsolution of (2.2),

$$
\zeta_{t}+(u \cdot \nabla) \zeta \leqslant 0 \text { at }\left(t_{0}, x_{0}\right)
$$

The Schwarz inequality now yields

$$
\zeta_{t}-L|\nabla \zeta| \leqslant \zeta_{t}+(u \cdot \nabla) \zeta \leqslant 0 \text { at }\left(t_{0}, x_{0}\right)
$$

so $\psi$ is a viscosity subsolution of $(2.4)$ on $Q$.

Lemma 2.2. Suppose that $u \in C(\bar{Q})$ and $a \in C(\mathrm{~T})$. There are unique largest and smallest solutions $\lambda$ and $\sigma$ of (2.2)-(2.3) which are bounded on every compact set in $\bar{Q}$. Moreover, $\lambda$ and $\sigma$ are expressed as

$$
\begin{aligned}
& \lambda(t, x)=\sup \{\psi(t, x) ; \psi \text { is a subsolution of (2.2)-(2.3)\}} \\
& \sigma(t, x)=\inf \{\psi(t, x) ; \psi \text { is a supersolution of }(2.2)-(2.3)\}
\end{aligned}
$$

Proof. Let $\Lambda$ denote the right hand side of (2.6). As well known there is a unique viscosity solution $\psi^{+}$(resp. $\psi^{-}$) of (2.4) (resp. (2.5)) with (2.3). By Proposition $2.1 \psi^{+}$ and $\psi^{-}$are, respectively, super- and subsolution of (2.2)-(2.3). Also any subsolution $\psi$ of (2.2)-(2.3) is a subsolution of (2.4)-(2.3) so a comparison theorem for (2.4) yields $\psi \leqslant \psi^{+}$. By Perron's method (cf. [Ish]) we see $\Lambda$ is a solution of (2.2)-(2.3) with

$$
\psi^{-} \leqslant \Lambda \leqslant \psi^{+}
$$

Since $\psi^{ \pm}$is continuous on $\bar{Q}, \Lambda$ is bounded on every compact set in $\bar{Q}$. The solution $\Lambda$ is a unique largest solution $\lambda$ because otherwise there would exist a solution $\varphi$ of (2.2)-(2.3) 
which is not smaller than $\Lambda$ and this contradicts the definition of $\Lambda$. We thus proved all statements on $\lambda=\Lambda$. The proof for $\sigma$ is completely parallel, so is omitted.

Lemma 2.3 (Uniqueness of level sets). Let $\lambda$ and $\sigma$ be, respectively, the largest and smallest solutions of (2.2)-(2.3). Let

$$
\begin{aligned}
& \Omega_{+}=\left\{(t, x) \in[0, T) \times \mathbf{T} ; \sigma_{*}(t, x)>0\right\} \\
& \Omega_{-}=\left\{(t, x) \in[0, T) \times \mathbf{T} ; \lambda^{*}(t, x)<0\right\}
\end{aligned}
$$

where $\lambda^{*}=-(-\lambda)_{*}$. The set $\Omega_{+}$(resp. $\left.\Omega_{-}\right)$is completely determined by the initial data $\Omega_{+}(0)$ (resp. $\Omega_{-}(0)$ ) and $u$, and is independent of choice of $a$.

Proof. Suppose that $a_{i} \in C(T)(i=1,2)$ satisfies

$$
\Omega_{+}(0)=\left\{x \in T ; a_{i}(x)>0\right\}
$$

Let $\sigma_{i}$ denote the smallest solution of (2.2)-(2.3) with $a=a_{i}$. We first take $\theta \in C(\mathbf{R})$ (strictly) increasing with $\theta(0)=0$ and $a_{1} \leqslant \theta\left(a_{2}\right)$. Such a function $\theta$, of course, exists (cf. [CGG1, Lemma 7.2]). Since the equation (2.2) is geometric, $\varphi:=\theta\left(\sigma_{2}\right)$ is a solution of (2.2)-(2.3) with $a=\theta\left(a_{2}\right)$ (cf. [CGG1, Theorem 5.2] or [CGG2, Theorem 2.3]). Moreover $\varphi$ is the smallest solution of (2.2)-(2.3) with $a=\theta\left(a_{2}\right)$ since $\theta$ and $\theta^{-1}$ preserve the order in $\mathbf{R}$.

We next observe that $\sigma_{1} \leqslant \varphi$. Indeed, $\psi=\min \left(\sigma_{1}, \varphi\right)$ is a supersolution of (2.2)-(2.3) with $a=a_{1}$ (cf. [CGG1, Proposition 2.2]). If $\sigma_{1} \leqslant \varphi$ were not true, there would exist $(t, x) \in Q$ such that $\psi(t, x)<\sigma_{1}(t, x)$. This contradicts the representation (2.7) of the smallest solution $\sigma_{1}$.

The inequality $\sigma_{1} \leqslant \varphi$ yields

$$
\left\{(t, x) ; \sigma_{1 *}(t, x)>0\right\} \subset\left\{(t, x) ; \sigma_{2 *}(t, x)>0\right\}
$$


If we choose $\theta$ so that $a_{2} \leqslant \theta\left(a_{1}\right)$, the other side inclusion also holds, so $\Omega_{+}$is completely determined by $\Omega_{+}(0)$.

The proof for $\Omega_{-}$is parallel, so is omitted.

Remark. Evans and Souganidis [ESou, Theorem 7.1] proved the uniqueness of level sets in $R^{n}$ when the equation (2.2) is

$$
u_{t}+H(x, \nabla u)=0
$$

where $H: \mathbf{R}^{n} \times \mathbf{R}^{n} \longrightarrow \mathbf{R}$ is uniformly Lipschitz, and positively homogeneous of degree one in the second variable. In this case there is no need to consider largest and smallest solutions because solutions of (2.10) with (2.3) are unique by comparison. The proof given there is different from those in [CGG1, 2] and does not seem to apply to second order equations. Of course the proof in [CGG1,2] does apply to second order equations.

Generalized evolution. Let $\Omega_{+}$(resp. $\Omega_{-}$) be an open sets in $M$. We say $\Omega_{+}$(resp. $\left.\Omega_{-}\right)$is a $+($resp. -$)$generalized evolution with speed $u \in C(\bar{Q})$ and initial data $\Omega_{+}(0)$ (resp. $\Omega_{-}(0)$ ) on interval $[0, T)$ if there is the smallest (resp. largest) solution $\sigma$ (resp. $\lambda$ ) of (2.2)-(2.3) with some $a \in C(\mathbf{T})$ satisfying (2.8) (resp. (2.9)).

Note that each level set of solutions of (2.2)-(2.3) independently moves by (2.1) at least formally. The \pm depends on the orientation of the interface.

For a given open set $\Omega_{+_{0}}$ in $T$ there is $a \in C(T)$ satisfying $\Omega_{+_{0}}=\{x ; a(x)>0\}$, so Lemmas 2.2 and 2.3 yield:

Theorem 2.4. For a given open set $\Omega_{+0}\left(\right.$ resp. $\left.\Omega_{-0}\right)$ in $\mathrm{T}$ there is a unique + (resp. - ) generalized evolution $\Omega_{+}$(resp. $\Omega_{-}$) with speed $u \in C(\bar{Q})$ and initial data $\Omega_{ \pm}(0)=\Omega_{ \pm 0}$ on $[0, T)$. If $\Omega_{+_{0}}$ and $\Omega_{-0}$ are disjoint, so are $\Omega_{+}$and $\Omega_{-}$.

THEOREM 2.5 (Stability). Let $\Omega_{+j}$ be the + generalized evolution with speed $u_{j} \in C(\bar{Q})$ and initial data $\Omega_{+j}(0)=\Omega_{+0}$ on $[0, T)$, where $j=1,2, \cdots$ and $Q=(0, T) \times \mathbf{T}$. Suppose that $T<\infty$ and $u_{j} \longrightarrow u$ in $C(\bar{Q})$ as $j \rightarrow \infty$. Let $\Omega_{+}$be the + generalized evolution on 
$[0, T)$ with speed $u$ and $\Omega_{+}(0)=\Omega_{+0}$. Let $K$ be a compact set in $\Omega_{+}$. Then $K$ is also contained in $\Omega_{+j}$ for sufficiently large $j$. The same holds for - evolution.

Proof. Let $\sigma_{j}$ be the smallest solution of

$$
\psi_{t}+\left(u_{j} \cdot \nabla\right) \psi=0, \quad \psi(0, x)=a(x) \in C(\mathbf{T})
$$

with $\Omega_{+_{0}}=\{x ; a(x)>0\}$. By the stability result of Barles and Perthame [BP, Appendix] the function

$$
\varphi(t, x):=\lim . \sigma_{j}(t, x):=\lim _{\substack{j \rightarrow \infty \\ c \downarrow 0}} \inf \left\{\sigma_{j}(s, y) ;|t-s|<\varepsilon,|y-x|<\varepsilon\right\}
$$

is a viscosity supersolution of (2.2) on $Q$ since $u_{j} \rightarrow u$ in $C(\bar{Q})$. Let $L$ be a constant such that $\sup _{Q}\left|u_{j}\right| \leqslant L$ for all $j$. We take a continuous solution $\psi^{+}$(resp. $\psi^{-}$) of (2.4) (resp. (2.5)) with (2.3). As in the proof of Lemma 2.2 , we have $\psi^{-} \leqslant \sigma_{j} \leqslant \psi^{+}$. This implies that $\psi^{-} \leqslant \varphi \leqslant \psi^{+}$on $[0, T) \times \mathbf{T}$, so we have $\varphi_{*}(0, x)=a(x)$. Therefore $\varphi$ is a supersolution of (2.2)-(2.3). Let $\sigma$ be the smallest solution of (2.2)-(2.3) so that $\varphi \geqslant \sigma$ by (2.7). For any compact set $K \subset \Omega_{+}$there is $\delta>0$ such that $\inf _{K} \sigma_{*} \geqslant \delta$ since $\sigma_{*}$ is lower semicontinuous. Since $\varphi \geqslant \sigma$ and $K$ is compact we see $\inf f_{K} \sigma_{j \star} \geqslant \delta / 2$ for sufficiently large $j$. This implies $K \subset \Omega_{+j}$ for large $j$. The proof for - evolution is parallel, so is omitted.

3. Global existence of weak solutions. We introduce a weak formulation of the problem (1.1)-(1.6) on T. Let $\Omega_{ \pm}$be two disjoint open sets in $[0, T) \times \mathbf{T}$. Let $\nu$ be a step function such that $\nu=\nu_{ \pm}$in $\Omega_{ \pm}$and $\nu=\left(\nu_{+}+\nu_{-}\right) / 2$ outside $\Omega_{+} \cup \Omega_{-}$where $0<\nu_{-}<\nu+$. Let $f$ be a tensor field on $Q=(0, T) \times \mathrm{T}$ such that $f=f_{ \pm}$on $\Omega_{ \pm}$. For given $\Omega_{ \pm}$we say vector field $u$ is a weak solution of (1.2)-(1.5) in $Q$ if $u \in C(\bar{Q})$ with $\nabla u \in L^{q}(Q)$ (for some $1<q<\infty$ ) and it solves

$$
u_{t}-\nabla \cdot(\nu D(u))+\nabla \pi=\nabla \cdot f+\nabla \cdot g, \nabla \cdot u=0, \quad \text { in } \quad Q=(0, T) \times \mathbf{T}
$$

in the sense of distribution with some $\pi$ and $g$ whose support spt $g$ is contained in $\Gamma=$ $\bar{Q} \backslash\left(\Omega_{+} \cup \Omega_{-}\right)$. By $L^{p}(Q)$ we mean the space of all periodic (in space) function $f$ on $(0, T) \times \mathbf{R}^{n}$ with period $\alpha=\left(\alpha_{1}, \cdots, \alpha_{n}\right)$ such that $\left.f\right|_{(0, T) \times R} \in L^{p}((0, T) \times R)$. 
If the Lebesgue measure of the interface $\Gamma$ is zero, then (3.1) yields (1.2)-(1.3) by interpreting $u=u_{ \pm}$in $\Omega_{ \pm}$. If $\{\Gamma(t)\}_{t \geq 0}$ is a smooth family of smooth surfaces, the boundary condition (1.5) is contained in (3.1). The condition (1.4) is automatic since $u \in C(\bar{Q})$.

We now state our main result in this paper.

Theonem 3.1. Let $p>2(n+1)$. Assume that $\Omega_{ \pm 0}$ are two disjoint open sets in $T$ and that $f \in L^{p}(Q)$ is a tensor field. Then there exists a positive constant $\delta=\delta(n, p)$ such that if

$$
\frac{\nu_{+}-\nu_{-}}{\nu_{+}}<\delta
$$

then there exists a weak solution $u \in C(\bar{Q})$ with $\nabla u \in L^{p}(Q)$ of (1.2)-(1.5) with (1.6) for generalized evolutions $\Omega_{ \pm} \subset \bar{Q}$ with initial data $\Omega_{ \pm 0}$ such that the speed of $\Omega_{ \pm}$equals $u$. Moreover $g$ in (3.1) can be taken as an element of $L^{p}\left(\left(0, T_{0}\right) \times T\right)$ for finite $T_{0} \leq T$. Here $T$ is allowed to be infinte.

4. Upper semicontinuous convexification. This section establishes a crucial abstract theory for (set-valued) mappings so that we apply Kakutani's fixed point theory. For this purpose we extend a mapping to an upper semicontinuous convex set-valued mapping.

For a given subset $A$ of a vector space $X$ let $\operatorname{co} A$ denote the convex hull of $A$, i.e.,

$$
\operatorname{co} A=\{t x+(1-t) y ; x, y \in A, 0 \leqslant t \leqslant 1\}
$$

Let $X$ and $Y$ be a normed space and a Banach space equipped with norms $\|\cdot\| x$ and $\left\|_{\text {. }}\right\|_{Y}$, respectively. For a set-valued mapping $S: X \longrightarrow 2^{Y}$ we define $S_{\varepsilon}: X \longrightarrow 2^{Y}$ by

$$
S_{e}(u)=\bigcup\left\{S(w) ;\|u-w\|_{X}<\varepsilon\right\} \subset Y
$$


for $u \in X$ and $\varepsilon>0$. Here $2^{Y}$ denotes the family of all subsets of $Y$. We introduce another set-valued mapping $\mathcal{S}: X \longrightarrow 2^{Y}$ defined by

$$
\mathcal{S}(u)=\bigcap_{c>0} \overline{\operatorname{coS}(u)}, \quad u \in X,
$$

where $\bar{B}$ denotes the closure of $B \subset Y$. In this paper we call $\mathcal{S}$ upper semicontinuous convexification of $S$ since it has the following properties;

LEMMA 4.1. (i) For each $u \in X$ the set $\mathcal{S}(u)$ is closed and convex in $Y$.

(ii) The mapping $\mathcal{S}$ is upper semicontinuous. In other words, if $u_{j} \longrightarrow u$ in $X, v_{j} \in \mathcal{S}\left(u_{j}\right)$ and $v_{j} \longrightarrow v$ in $Y$, then $v \in \mathcal{S}(u)$.

(iii) If $\mathcal{S}(u)$ is nonempty for all $u \in X$, so is $\mathcal{S}$.

Proof. (i) Clearly, $\mathcal{S}(u)$ is closed. Since the closure of a convex set is convex and the intersection of a family of convex sets is still convex, we see $\mathcal{S}(u)$ is convex.

(ii) Suppose that $v \notin \mathcal{S}(u)$. Then there would exist $\delta>0$ such that

$$
v \notin A_{\delta}(u):=\overline{\operatorname{co} S_{\delta}(u)}
$$

Since $A_{\delta}(u)$ is closed, there would exist $k$ such that $j \geqslant k$ implies that $v_{j} \notin A_{\delta}(u)$. Since $u_{j} \longrightarrow u$ we may asssume that $\left\|u_{j}-u\right\|_{X}<\delta / 2$ for $j \geqslant k$ by taking $k$ larger. By the definition of $S_{e}$ we see

$$
A_{\delta}(u) \supset A_{\delta / 2}\left(u_{j}\right), \quad j \geqslant k
$$

This inclusion now would imply $v_{j} \notin A_{\delta / 2}\left(u_{j}\right)$, i.e., $v_{j} \notin \mathcal{S}\left(u_{j}\right)$ for $j \geqslant k$, which leads a contradiction.

(iii) Since $S_{e}(u)$ containes $S(u)$, so does $S(u)$.

We have introduced the upper semicontinuous convexification so that we apply Kakutani's fixed point theory. We state an easy consequence of this fixed point theory for later use.

Proposition 4.2. Let $K$ be a convex compact subset of a Banach space $X$ and let $S: X \longrightarrow 2^{K} \subset 2^{X}$ be a nonempty set-valued mapping. Let $\mathcal{S}$ be the upper semicontinuous convexification of $S$. Then $\mathcal{S}$ has a fixed point $\bar{u} \in K \cap \mathcal{S}(\bar{u})$. 
Proof. Since $K$ is convex and closed, values of $\mathcal{S}$ are contained in $K$. By Lemma 4.1 we see $\mathcal{S}$ is an upper semicontinuous set-valued mapping $X \longrightarrow 2^{K}$ with nonempty closed convex values. The existence of a fixed point of $\mathcal{S}$ now follows from Kakutani's fixed point theorem $[\mathrm{AF}]$.

\section{Stokes equations with discontinuous coefficients.}

Let us recall anisotropic Sobolev spaces of fractional orders (cf. [Ya, Example 1.1 and Section 3] and [Tri, Section 2.13]) though our notation differs from them. For $1<p<\infty$ and $0<s<\infty$, let $H_{p}^{s, 2 s}=H_{p}^{s, 2 s}\left(\mathbf{R} \times \mathbf{R}^{n}\right)$ denote

$$
H_{p}^{d, 2,}=\left\{f \in L^{p}\left(\mathbf{R} \times \mathbf{R}^{n}\right) ;\|f\|_{H_{p}^{;, 2 s}}=\left\|\mathcal{F}^{-1}\langle\tau, \xi\rangle^{2,} \mathcal{F} f\right\|_{L^{\prime}\left(\mathbf{R} \times \mathbf{R}^{n}\right)}<\infty\right\}
$$

with $\langle\tau, \xi\rangle=\left[\left\{1+|\xi|^{2}+\left(1+2|\xi|^{2}+|\xi|^{4}+4 \tau^{2}\right)^{1 / 2}\right\} / 2\right]^{1 / 2}$. Here $(\mathcal{F} f)(\tau, \xi)$ denotes the Fourier transform of $f(t, x)$ on $\mathbf{R} \times \mathbf{R}^{n} \ni(t, x)$. The multiplier $\langle\tau, \xi\rangle$ is actually the unique positive root $t$ of

$$
t^{-2}+t^{-2 \cdot 2} \tau^{2}+t^{-2}|\xi|^{2}=1
$$

For a domain $D$ in $\mathbf{R}^{n+1}$ let $H_{p}^{\prime, 2,}(D)$ denote the space of all $f \in L^{p}(D)$ which can be extended to an element $\bar{f}$ of $H_{p}^{\prime, 2 s}$. The space $H_{p}^{\prime, 2,}(D)$ is equipped with the norm

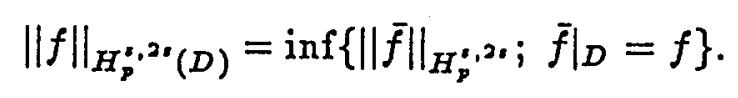

Let $H_{p}^{s, 2 s}(Q)$ denote the space of all periodic (in space) functions $f$ defined on $(0, T) \times \mathbf{R}^{n}$ with the period $\alpha=\left(\alpha_{1}, \cdots, \alpha_{n}\right)$ such that

$$
\left.f\right|_{(0, T) \times R} \in H_{p}^{j, 2 \prime}((0, T) \times R) .
$$

The space $H_{p}^{\prime, 2 s}(Q)$ is equipped with the norm

$$
\|f\|_{H_{p}^{\prime, 2 s}(Q)}=\|f\|_{H_{p}^{s, 20}((0, T) \times R)} .
$$

We shall write $H_{p}^{1 / 2,1}(Q)$ simply by $\mathcal{H}_{p}(Q)$. We begin with an a priori estimates for the heat equation. 
LEMMA 5.1. Let $0<T \leqslant \infty$ and $Q=(0, T) \times \mathbf{T}$. Let $2<p<\infty$ and let $F \in L^{p}(Q)$ be a vector field. Then there exists a unique solution $u \in \mathcal{H}_{p}(Q)$ of

$$
\begin{aligned}
& u_{t}-\Delta u=\nabla \cdot F, \quad \text { in } Q, \\
& \left.u\right|_{t=0}=0 .
\end{aligned}
$$

Moreover there is a constant $C_{1}=C_{1}(n, p)$ such that

$$
\|u\|_{\mathcal{H}_{p}(Q)} \leqslant C_{1}\|F\|_{L p(Q)}
$$

The restriction $2<p$ guarantees that $u \in \mathcal{H}_{p}(Q)$ has a trace at $t=0$. However if we interpret $\left.u\right|_{t=0}=0$ in a suitable way, the restriction $p>2$ is weakened as $p>1$.

Proof. The uniqueness is standard. For example, multiplying $u$ with $u_{t}-\Delta u=0$ and integrating in space by parts yields a differential inequality which implies $u \equiv 0$.

We extend $u$ and $F$ periodically outside $R$ so that $u$ solves

$$
u_{t}-\Delta u=\nabla \cdot F, \quad \text { in }(0, T) \times \mathbf{R}^{n} .
$$

The solution $u$ is expressed as

$$
u(t, x)=\int_{0}^{t} \int_{\mathbf{R}^{n}}(\nabla g)(t-s, x-y) \cdot F(y) d y d s,
$$

where $g(t, x)=(4 \pi t)^{-n / 2} \exp \left(-|x|^{2} / 2\right)$ is the heat kernel. Since $F$ is periodic with period $\alpha=\left(\alpha_{1}, \cdots, \alpha_{n}\right)$, we observe that

$$
u(t, x)=\sum_{\sigma \in \mathbf{Z}^{n}} v(t, x-\sigma \alpha), \quad v(t, x)=\int_{0}^{t} \int_{R}(\nabla g)(t-s, x-y) \cdot F(y) d y d s,
$$

where $\sigma \alpha=\left(\sigma_{1} \alpha_{1}, \cdots, \sigma_{n} \alpha_{n}\right)$. Note that

$$
\|u\|_{\mathcal{H}_{p}(Q)} \leq \sum_{\sigma \in \mathbf{Z}^{n}}\|v(\cdot, \cdot-\sigma \alpha)\|_{\left.H_{p}^{1 / 2,1}((0, T) \times R)\right)} \leq\|v\|_{H_{p}^{1 / 2,1}(D)}
$$

with $D=(0, T) \times \mathbf{R}^{n}$. By Mikhlin's lemma (cf. [MS]) we see

$$
\|v\|_{H_{p}^{1 / 2,1}(D)} \leq C_{1}\|F\|_{L^{P}(Q)}
$$

with $C_{1}=C_{1}(n, p)$. These two inequalities yield Lemma 5.1.

We apply Lemma 5.1 and a perturbation argument (cf. [Cam], [GY]) to the Stokes system with discontiuous coefficients: 
Proposition 5.2. Assume that $0<T \leqslant \infty$ and $2<p<\infty$. Assume that $\nu \in L^{\infty}(Q)$ satisfies

$$
0<\nu_{-} \leq \nu \leq \nu_{+}
$$

with some constants $\nu_{ \pm}$. Let $f \in L^{p}(Q)$ be a tensor field. Then there exists a positive constant $\delta=\delta(n, p)$ such that

$$
\frac{\nu_{+}-\nu_{-}}{\nu_{+}}<\delta
$$

implies that the Stokes system

$$
\begin{aligned}
& u_{t}-\nabla \cdot(\nu D(u))+\nabla \pi=\nabla \cdot f, \nabla \cdot u=0, \text { in } Q, \\
& \left.u\right|_{t=0}=0
\end{aligned}
$$

has a unique solution $u \in \mathcal{H}_{p}(Q)$ (with some function $\pi$ ) satisfying

$$
\|u\|_{\mathcal{H}_{p}(Q)} \leqslant \frac{C_{2}}{\nu_{+}}\|f\|_{L^{p}(Q)}
$$

with $C_{2}=C_{2}(n, p)$.

Proof. Let $P$ be the projection of $L^{p}(\mathrm{~T})$ to $L_{\sigma}^{p}(\mathrm{~T})$ associated with the Helmholtz decomposition

$$
\begin{gathered}
L^{p}(\mathbf{T})=L_{\sigma}^{p}(\mathbf{T}) \oplus\left\{\nabla \pi \in L^{p}(\mathbf{T}) ; \pi \in L^{p}(\mathbf{T})\right\} \\
L_{\sigma}^{p}(\mathbf{T})=\left\{u \in L^{p}(\mathbf{T}) ; \nabla \cdot u=0 \text { in } \mathbf{T}\right\}
\end{gathered}
$$

Since $\mathrm{T}$ has no boundary, $P$ commutes with partial derivatives on $\mathrm{T}$. Applying $P$ to the first equation of (5.3) yields

$$
u_{t}-\nabla \cdot(P \nu D(u))=\nabla \cdot(P f) .
$$

Here $P f$ is a tensor field defined by

$$
(P f)_{i j}=\left(P f_{j}\right)_{i} \quad 1 \leq i, j \leq n
$$


for a tensor field $f$ and $f_{j}$ represents a vector field defined by $f_{j}=\left(f_{i j}\right)_{1 \leq i \leq n}$. From (5.5) it follows

$$
u_{t}-\nu_{+} \Delta u=\nabla \cdot P\left(f+\left(\nu-\nu_{+}\right) D(u)\right)
$$

since $\nabla \cdot u=0$.

We shall solve (5.6) with $\left.u\right|_{t=0}=0$ by a successive approximation. Let $u_{j+1}$ be a solution of

$$
\begin{aligned}
& \partial_{t} u_{j+1}-\nu_{+} \Delta u_{j+1}=\nabla \cdot P\left(f+\left(\nu-\nu_{+}\right) D\left(u_{j}\right)\right) \\
& \left.u_{j+1}\right|_{t=0}=0
\end{aligned}
$$

for $j \geq 1$ and let $u_{1} \equiv 0$. Since $P$ is bounded from $L^{p}(\mathrm{~T})$ to $L_{\sigma}^{p}(\mathrm{~T})$ and $\|D(u)\|_{L^{p}(Q)} \leq$ $2 C_{0}\|u\|_{\mathcal{H}_{p}(Q)}$ (cf. Appendix, Lemma A.1(vi)), it follows from (5.1) that

$$
\left\|P\left(f+\left(\nu-\nu_{+}\right) D\left(u_{j}\right)\right)\right\|_{L^{P}(Q)} \leq C\left(\|f\|_{L^{P}(Q)}+2 C_{0}\left(\nu_{+}-\nu_{-}\right)\left\|u_{j}\right\|_{\mathcal{H}_{p}(Q)}\right)
$$

The bound $C$ of $P$ here is actually independent of $\mathbf{T}$. Indeed, note that $P u=u-\nabla q$ with $\Delta q=\nabla \cdot u$ in $T$. Extend $q$ and $u$ periodically outside $R$ so that $\Delta q=\nabla \cdot u$ is regarded as an equation on $R^{n}$. As in the proof of Lemma 5.1, applying Mikhlin's lemma to the integral representation of $\nabla q$ we obtain

$$
\|\nabla q\|_{L^{P}(R)} \leq C^{\prime}\|u\|_{L^{P}(R)}
$$

with $C^{\prime}=C^{\prime}(n, p)$

Applying Lemma 5.1 with a change of a variable $s=t / \nu_{+}$to $(5.7)$, we now obtain

$$
\begin{aligned}
& \left\|u_{j+1}\right\|_{\mathcal{H}_{p}(Q)} \leqslant \frac{C_{1} C}{\nu_{+}}\|f\|_{L^{p}(Q)}+C^{\prime \prime} \frac{\nu_{+}-\nu_{-}}{\nu_{+}}\left\|u_{j}\right\|_{\mathcal{H}_{p}(Q)} \\
& C^{\prime \prime}=2 C_{0} C_{1} C .
\end{aligned}
$$

We thus observe that $u_{j} \in \mathcal{H}_{p}(Q)$ for all $j \geq 1$. Choose $\delta$ such that $C^{\prime \prime} \delta<1 / 2$. Since the equation (5.7) is linear in $u_{j+1}$ and $u_{j}$, the difference $w_{j+1}=u_{j+1}-u_{j}$ solves

$$
\begin{aligned}
& \partial_{t} w_{j+1}-\nu_{+} \Delta w_{j+1}=\nabla \cdot P\left(\left(\nu-\nu_{+}\right) D\left(w_{j}\right)\right), \text { in } Q \\
& \left.w_{j+1}\right|_{t=0}=0
\end{aligned}
$$


As in deriving (5.8) applying Lemma 5.1 we observe, by (5.2), that

$$
\begin{aligned}
\left\|w_{j+1}\right\|_{\mathcal{H}_{p}(Q)} & \leqslant C^{\prime \prime} \frac{\nu_{+}-\nu_{-}}{\nu_{+}}\left\|w_{j}\right\|_{\mathcal{H}_{p}(Q)} \\
& \leq \frac{1}{2}\left\|w_{j}\right\|_{\mathcal{H}_{p}(Q)}
\end{aligned}
$$

for $j \geq 2$. This implies that $\left\{u_{j}\right\}$ is a Cauchy sequence in $\mathcal{H}_{p}(Q)$. The limit $u$ of $\left\{u_{j}\right\}$ solves (5.6) with $\left.u\right|_{t=0}=0$. The estimate (5.8) yields

$$
\|u\|_{\mathcal{H}_{p}(Q)} \leqslant \frac{C_{1} C}{\nu_{+}}\|f\|_{L^{p}(Q)}+\frac{1}{2}\|u\|_{\mathcal{H}_{p}(Q)} .
$$

We now obtain (5.4). If $u$ solves (5.6), then $\nabla \cdot u=0$ since $P$ commutes with partial derivatives. We have thus constructed a solution $u \in \mathcal{H}_{p}(Q)$ of (5.3) with (5.4) under (5.2). The uniqueness of solutions follows from (5.4).

6. Proof of Theorem 3.1. Assume that $0<T<\infty$. For $u \in C(\bar{Q})$ let $\Omega_{ \pm} \subset \bar{Q}$ be generalized evolutions with speed $u$ and initial data $\Omega_{ \pm 0}$. Let $\nu=\nu_{u}$ be a step function such that $\nu=\nu_{ \pm}$in $\Omega_{ \pm}$and $\nu=\left(\nu_{+}+\nu_{-}\right) / 2$ outside $\Omega_{+} \cup \Omega_{-}$with $0<\nu_{-}<\nu_{+}$. Assume that $f \in L^{p}(Q)$. If positive constant $\delta$ is chosen as in Proposition 5.2, then there is a unique solution $\tilde{u}$ of (5.3) for $\nu=\nu_{u}$ such that

$$
\tilde{u} \in K=\left\{u \in \mathcal{H}_{p}(Q) ;\|u\|_{\mathcal{H}_{p}(Q)} \leqslant \frac{C_{2}}{\nu_{+}}\|f\|_{L^{P}(Q)}\right\} .
$$

We define a mapping $S: C(\bar{Q}) \longrightarrow 2^{K}$ by $S(u):=\{\tilde{u}\}$. If $p>2(n+1)$, the inclusion

$$
\mathcal{H}_{p}(Q) \subset C^{\mu}(\bar{Q})
$$

for $\mu=1 / 2(n+1)-1 / p$ is continuous (see Appendix) and Ascoli-Arzela's theorem implies that $K$ is compact in Banach space $C(\bar{Q})$ since $T<\infty$. Unfortunately Leray-Schauder's fixed point theory does not apply to $S$ since $S$ may not be continuous. We consider the upper semicontinuous convexification $S$ of $S$ in Section 4.

LEMMA 6.1. Let $\mathcal{S}$ be the upper semicontinuous convexification of $S$. If $v \in \mathcal{S}(u)$, then $v$ is a weak solution of (1.2)-(1.5) with (1.6) for generalized evolutions $\Omega_{ \pm}$with speed $u$ and the initial data $\Omega_{ \pm 0}$ Moreover $g$ in (3.1) belongs to $L^{p}(Q)$. 
Proof. By the definition of $\mathcal{S}$, if $v \in \mathcal{S}(u)$, then for each $k=1,2, \cdots$, there is a sequence $\left\{v_{m}^{k}\right\}_{m=1}^{\infty}$ converging to $v$ in $C(\bar{Q})$ such that $v_{m}^{k} \in \operatorname{co} S_{1 / k}(u)$, i.e., $v_{m}^{k}$ is expressed as

$$
v_{m}^{k}=\sum_{j=m}^{\ell} \lambda_{j}^{m k} \tilde{u}_{j}^{k}, \quad\left\{\tilde{u}_{j}^{k}\right\}=S\left(u_{j}^{k}\right), \quad \ell=\ell(m, k),
$$

with some $\lambda_{j}^{m k}$ and $u_{j}^{k} \in C(\bar{Q})$ such that

$$
\sum_{j=m}^{L} \lambda_{j}^{m k}=1, \quad \lambda_{j}^{m k} \geqslant 0, \quad\left\|u_{j}^{k}-u\right\|_{C(\bar{Q})}<1 / k
$$

for all $k \geq 1$. By a diagonal argument there are a sequence $u_{j}$ converging to $u$ in $C(\bar{Q})$ and $\lambda_{j}^{m}, \cdots, \lambda_{j}^{\ell_{m}}$ with

$$
\sum_{j=m}^{\ell_{m}} \lambda_{j}^{m}=1, \quad \lambda_{j}^{m} \geqslant 0
$$

such that

$$
v_{m}=\sum_{j=m}^{\iota_{m}} \lambda_{j}^{m} \tilde{u}_{j}, \quad\left\{\tilde{u}_{j}\right\}=S\left(u_{j}\right)
$$

converges to $v$ in $C(\bar{Q})$ as $m \rightarrow \infty$. By the definition of $S, \tilde{u}_{j}$ solves

$$
\begin{aligned}
& \partial_{t} \tilde{u}_{j}-\nabla \cdot\left(\nu_{u_{j}} D\left(\tilde{u}_{j}\right)\right)+\nabla \tilde{\pi}_{j}=\nabla \cdot f, \quad \text { in } Q, \\
& \nabla \cdot \tilde{u}_{j}=0, \quad \text { in } Q, \\
& \left.\tilde{u}_{j}\right|_{t=0}=0,
\end{aligned}
$$

with some $\tilde{\pi}_{j}$. Multiplying $\lambda_{j}^{m}$ and adding from $m$ to $l_{m}$ we see

$$
\begin{aligned}
& \partial_{t} v_{m}-\nabla \cdot\left(\nu_{u} D\left(v_{m}\right)\right)+\nabla \pi_{m}=\nabla \cdot f+\nabla \cdot g_{m}, \quad \text { in } Q, \\
& \nabla \cdot v_{m}=0, \quad \text { in } Q \\
& \left.v_{m}\right|_{t=0}=0,
\end{aligned}
$$

with

$$
\begin{aligned}
& \pi_{m}=\sum_{j=m}^{\ell_{m}} \lambda_{j}^{m} \tilde{\pi}_{j}, \\
& g_{m}=\sum_{j=m}^{\ell_{m}} \lambda_{j}^{m}\left(\nu_{u_{j}}-\nu_{u}\right) D\left(\tilde{u}_{j}\right) .
\end{aligned}
$$


Since $K$ is convex and bounded, the sequence $\left\{v_{m}\right\}$ is bounded in $\mathcal{H}_{p}(Q)$. We thus observe that $D\left(v_{m}\right) \rightarrow D(v)$ weakly in $L^{p}(Q)$ since $v_{m} \rightarrow v$ in $C(\bar{Q})$. Since $\tilde{u}_{j} \in K$, the sequence $\left\{g_{m}\right\}$ is bounded in $L^{p}(Q)$. Taking a subsequence if necessary, $g_{m} \rightarrow g$ weakly in $L^{p}(Q)$ for some $g \in L^{p}(Q)$. Letting $m \rightarrow \infty$ in (6.1) yields

$$
\begin{aligned}
& \partial_{t} v-\nabla \cdot\left(\nu_{u} D(v)\right)+\nabla \pi=\nabla \cdot f+\nabla \cdot g, \quad \text { in } Q, \\
& \nabla \cdot v=0, \quad \text { in } Q, \\
& \left.v\right|_{t=0}=0,
\end{aligned}
$$

for some $\pi$.

It remains to prove that spt $g \subset \bar{Q} \backslash\left(\Omega_{+} \cup \Omega_{-}\right)$. Let $C$ be a compact set in $\Omega_{+} \cup \Omega_{-}$. Since $u_{j} \rightarrow u$ in $C(\bar{Q})$, we see, by Theorem $2.5, \nu_{u_{j}}=\nu_{u}$ on $C$ for sufficiently large $j$. This implies that $g_{j} \equiv 0$ on $C$ for sufficiently large $j$. Since $g_{j} \rightarrow g$ weakly in $L^{p}(Q)$ and $C$ can be taken as an arbitrary ball in $\Omega_{+} \cup \Omega_{-}$, we conclude that $g \equiv 0$ on $\Omega_{+} \cup \Omega_{-}$.

If $T<\infty$ and $p>2(n+1), K$ is compact and convex in $X=C(\bar{Q})$. By Proposition $4.2 \mathcal{S}$ has a fixed point $u \in K \cap \mathcal{S}(u)$. By Lemma 6.1 this $u$ is a desired weak solution in Theorem 3.1 .

To complete the proof of Theorem 3.1 it remains to construct a global solution in $(0, \infty)$. For $0<T<\infty$ we write $Q$ by $Q_{T}, K$ by $K_{T}$ and $\mathcal{S}$ by $\mathcal{S}_{T}$ to emphasize the dependence of $T$. For $T_{1}<T_{2}<\cdots<T_{i} \longrightarrow \infty$ let $u_{T_{i}}$ be a fixed point in $K_{T_{i}} \cap \mathcal{S}_{T_{i}}\left(u_{T_{i}}\right)$. Since $\delta$ in Lemma 5.2 is independent of time for each $T<\infty$, the restrictions $\left\{\hat{u}_{i}\right\}$ of $\left\{u_{T_{i}}\right\}$ on $t \leq T$ are bounded in $K_{T} \subset \mathcal{H}_{p}\left(Q_{T}\right)$ for sufficiently large $i$. Since the inclusion $\mathcal{H}_{p}\left(Q_{T}\right) \subset C\left(\overline{Q_{T}}\right)$ is compact for $p>2(n+1)$ and $T<\infty$, a diagonal argument yields a subsequence $\left\{\hat{u}_{i^{\prime}}\right\}$ and $w \in C((0, \infty) \times \mathbf{T})$ satisfying

$$
\hat{u}_{i \prime} \longrightarrow w \text { in } C\left(\overline{Q_{T}}\right)
$$

Since $\hat{u}_{i^{\prime}} \in \mathcal{S}_{T}\left(\hat{u}_{i^{\prime}}\right) \subset C\left(\overline{Q_{T}}\right)$ and since the graph of $\mathcal{S}_{T}: C\left(\overline{Q_{T}}\right) \longrightarrow 2^{C\left(\overline{Q_{T}}\right)}$ is closed in $C\left(\overline{Q_{T}}\right) \times C\left(\overline{Q_{T}}\right),(6.2)$ implies $\left.w\right|_{Q_{T}} \in \mathcal{S}_{T}\left(\left.w\right|_{Q_{T}}\right) \subset C\left(\overline{Q_{T}}\right)$. Since $T$ is arbitrary, this yields a desired global solution in $(0, \infty)$. 
7. Appendix. We list a couple of properties of anisotropic Sobolev spaces for the reader's convenience since such spaces are less familiar than isotropic ones.

LEMmA A.1. (i) For $0 \leq s \leq 1$ the space $H_{p}^{s, 2 s}\left(\mathbf{R}^{n+1}\right)$ is isomorphic to the complex interpolation space $\left[L^{p}\left(\mathbf{R}^{n+1}\right), H_{p}^{1,2}\left(\mathbf{R}^{n+1}\right)\right]$, as Banach spaces.

(ii) The norm $\|f\|_{H_{p}^{1,2}}$ is equivalent to the norm

$$
\|f\|_{L^{p}}+\left\|\nabla^{2} f\right\|_{L^{p}}+\left\|\partial_{t} f\right\|_{L^{p}}
$$

(iii) Let $D$ be a domain in $\mathbf{R}^{n+1}$ of the form $\left(t_{0}, t_{1}\right) \times \Omega$ with smoothly bounded domain $\Omega$ in $\mathbf{R}^{n}$. There is a continuous linear operator e from $H_{p}^{1,2}(D)$ to $H_{p}^{1,2}\left(\mathbf{R}^{n+1}\right)$ such that ef $=f$ on $D$.

(iv) $H_{p}^{s, 2 s}(D)=\left[L^{p}(D), H_{p}^{1,2}(D)\right]_{s}$.

(v) For $p>2(n+1)$ the space $H_{p}^{1 / 2,1}(Q)$ is continuously embedded in $C^{\mu}(\bar{Q})$ with $\mu=$ $1 / 2(n+1)-1 / p$

(vi) There is a constant $C_{0}=C_{0}(n, p)$ such that

$$
\left\|\frac{\partial u}{\partial x_{j}}\right\|_{L^{p}(Q)} \leq C_{0}\|u\|_{\mathcal{H}_{p}(Q)} \text { for all } u \in \mathcal{H}_{p}(Q), j=1, \cdots, n \text {. }
$$

Proof. (i) For $f \in H_{p}^{1,2}\left(\mathbf{R}^{n+1}\right)$ we set

$$
A f=\mathcal{F}^{-1}\langle\tau, \xi\rangle^{2} \mathcal{F} f
$$

The operator $A$ is closed in $L^{p}\left(\mathbf{R}^{n+1}\right)$ with the domain $\mathcal{D}(A)=H_{p}^{1,2}\left(\mathbf{R}^{n+1}\right)$. By Mikhlin's lemma the operator norm in $L^{p}$ of the pure imaginary power $A^{i y}$ is bounded by a constant multiple of $\mathrm{e}^{\gamma|y|}$ for some $\gamma>0$. A standard argument (see e.g. [GS, Section 6]) yields

$$
H_{p}^{\prime, 2 s}\left(\mathbf{R}^{n+1}\right)=\mathcal{D}\left(A^{s}\right)=\left[L^{p}\left(\mathbf{R}^{n+1}\right), H_{p}^{1,2}\left(\mathbf{R}^{n+1}\right)\right]_{。}
$$

(ii) We observe through Mikhlin's lemma that

$$
\begin{aligned}
\|A f\|_{L^{p}} & \leq C\left\|\left(\partial_{t}-\Delta+1\right) f\right\|_{L^{p}} \\
& \leq C\left(\left\|\partial_{t} f\right\|_{L^{p}}+\left\|\nabla^{2} f\right\|_{L^{p}}+\|f\|_{L^{p}}\right) \\
& \leq C\|A f\|_{L^{p}}
\end{aligned}
$$


(iii) We may assume $t_{0}=0$. As well-known there is a continuous extension $e_{1}$ : $H_{p}^{2}(\Omega) \rightarrow H_{p}^{2}\left(\mathrm{R}^{n}\right)$. For $f \in H_{p}^{1,2}(D)$ we set

$$
\tilde{f}(t, x)=\left\{\begin{array}{l}
\left(e_{1} f\right)(-t, x) \text { for }(t, x) \in\left(-t_{1}, 0\right) \times \mathbf{R}^{n} \\
\left(e_{1} f\right)\left(2 t_{1}-t, x\right) \text { for }(t, x) \in\left(t_{1}, 2 t_{1}\right) \times \mathbf{R}^{n}
\end{array}\right.
$$

so that $\tilde{f}$ is defined on $\left(-t_{1}, 2 t_{1}\right) \times \mathbf{R}^{n}$. We then take $\varphi \in C_{0}^{\infty}\left(\left(-t_{1}, 2 t_{1}\right) \times \mathbf{R}^{n}\right)$ so that $\varphi \equiv 1$ on $D$. By the characterization of $H_{p}^{1,2}$ norm in (ii) we observe that the operator ef $:=\varphi \tilde{f}$ is continuous from $H_{p}^{1,2}(D)$ to $H_{p}^{1,2}\left(\mathbf{R}^{n+1}\right)$. Clearly $e f=f$ on $D$.

(iv) Interpolating $e: H_{p}^{1,2}(D) \rightarrow H_{p}^{1,2}\left(\mathbf{R}^{n+1}\right)$ and $e: L^{p}(D) \rightarrow L^{p}\left(\mathbf{R}^{n+1}\right)$, we observe that $e$ is a bounded linear operator

$$
e:\left[L^{p}(D), H_{p}^{1,2}(D)\right]_{\iota} \rightarrow\left[L^{p}\left(\mathbf{R}^{n+1}\right), H_{p}^{1,2}\left(\mathbf{R}^{n+1}\right)\right]_{\iota}=H_{p}^{s, 2 \iota}\left(\mathbf{R}^{n+1}\right)
$$

Since the restriction $r: H_{p}^{s, 2 s}\left(\mathbf{R}^{n+1}\right) \rightarrow H_{p}^{s, 2,}(D)$ is continuous, there is a continuous inclusion from $\left[L^{p}(D), H_{p}^{1,2}(D)\right]$, to $H_{p}^{s, 2,}(D)$.

Interpolating $r: H_{p}^{1,2}\left(\mathbf{R}^{n+1}\right) \rightarrow H_{p}^{1,2}(D)$ and $r: L^{p}\left(\mathbf{R}^{n+1}\right) \rightarrow L^{p}(D)$, we observe that $H_{p}^{s, 2 s}(D)$ is continuously included in $\left[L^{p}(D), H_{p}^{1,2}(D)\right]$, since $r$ is surjective and the topology of $H_{p}^{s, 2 s}(D)$ is strongest such that $r$ is continuous. This proves the identity of (iv).

(v) We take $D=(0, T) \times \Omega$ such that $\Omega$ contains the closed rectangle $R$. For $f \in$ $H_{p}^{1 / 2,1}(Q)$ the mapping

$$
j:\left.f \mapsto f\right|_{D}
$$

is continuous from $H_{p}^{1 / 2,1}(Q)$ to $H_{p}^{1 / 2,1}(D)$ since $D$ is bounded.

Note that $H_{p}^{1,2}(D) \subset H_{p}^{1}(D)$ by (ii), where $H_{p}^{1}(D)$ denotes an isotropic $L^{p}$ Sobolev space of order one. By (iv) we observe that $H_{p}^{1 / 2,1}(D) \subset H_{p}^{1 / 2}(D)$ since

$$
H_{p}^{1 / 2}(D)=\left[L^{p}(D), H_{p}^{1}(D)\right]_{1 / 2}
$$

(cf. [Tr, 4.3.1]). The Sobolev inequality implies

$$
H_{p}^{1 / 2}(D) \subset C^{\mu}(\bar{D})
$$


with $\mu=1 / 2(n+1)-1 / p$ provided that $p>2(n+1)$ (see [Tr, p.327, 4.6.1]). Thus $H_{p}^{1 / 2,1}(D)$ is continuously embedded in $C^{\mu}(\bar{D})$. The mapping $j$ now gives a continuous mapping

$$
H_{p}^{1 / 2,1}(Q) \rightarrow C^{\mu}(\bar{D})
$$

such that

$$
j f=f \quad \text { on }(0, T) \times R .
$$

This implies that the inclusion

$$
H_{p}^{1 / 2,1}(Q) \subset C^{\mu}(\bar{Q})
$$

is continuous.

(vi) For $u \in \mathcal{H}_{p}(Q)$ let $v \in H_{p}^{1 / 2,1}$ be an extension of $u$ such that

$$
\|v\|_{H_{p}^{1 / 2,1}} \leq 2\|u\|_{\mathcal{H}_{p}(Q)}
$$

By Mikhlin's lemma we have

$$
\left\|\frac{\partial u}{\partial x_{j}}\right\|_{L p(Q)} \leq C\|v\|_{H_{p}^{1 / 2,1}, \quad j=1, \cdots, n}
$$

with $C=C(n, p)$. These two inequalities yield (vi).

\section{REFERENCES}

[Al] G. Allain, Small-time existence for the Navier-Stokes equations with a free surface, Appl. Math. Optim. 16 (1987), 37-50.

[AF] J. P. Aubin and H. Frankowska, "Set-valued Analysis," Birkhäuser, Boston-BaselBerlin, 1990.

[BP] G. Barles and B. Perthame, Discontinuous solutions of deterministic optimal stopping time problems, RAIRO modèl, Math. et Anal. Num. 21 (1987), 557-579.

[Be 1] J. T. Beale, The initial value problem for the Navier-Stokes equations with a free, surface, Comm. Pure Appl. Math. 34 (1981), 359-392. 
[Be 2] J. T. Beale, Large-time regularity of viscous surface waves, Arch. Rational Mech. Anal. 84 (1984), 307-352.

[Cam] S. Campanato, $L^{p}$ regularity for weak solutions of parabolic systems, Ann. Scuola Norm. Sup. Pisa 7 (1980), 65-85.

[CGG1] Y.-G. Chen, Y. Giga and S. Goto, Uniqueness and existence of viscosity solutions of generalized mean curvature flow equations, J. Differential Geometry, 33 (1991), 749-786.

[CGG2] —- Analysis toward snow crystal growth, Proc. of international symposium on Functional Analysis and Related topics (ed. S. Koshi), (1991), 43-57. World Scientific, Singapore-New Jersey-London-Hong Kong.

[CIL] M. G. Crandall, H. Ishii and P. L. Lions, User's guide to viscosity solutions of second order partial differential equations, Bull. Amer. Math. Soc.(to appear).

[De1] I. V. Denisova, A priori estimtes of the solution of a linear time-dependent problem connected with the motion of a drop in a fluid medium, Trudy Matem Inst. Steklov. 188 (1990), 1-24.

[De2] I. V. Denisova, A probrem on the motion of a drop in a fluid medium, preprint(in Russian).

[ES] L. C. Evans and J. Spruck, Motion of level sets by mean curvature I, J. Differential Geometry 33 (1991), 635-681.

[ESou] L. C. Evans and P. E. Souganidis, Differential games and representation formulas for solutions of Hamilton-Jacobi-Isaccs equations, Indiana Univ. Math. J. 33(1984), 773-797.

[GGI] Y. Giga, S. Goto and H. Ishii, Global existence of weak solutions for interface equations coupled with diffusion equations, SIAM J. Math. Anal. (to appear).

[GS] Y. Giga and H. Sohr, On the Stokes operator in exterior domains, J. Fac. Sci. Univ. Tokyo Sect. IA, Math. 36 (1989), 103-130.

[GY] Y. Giga and Z. Yoshida, A dynamic free-boundary problem in plasma physics, SIAM J. Math. Anal. 21 (1990), 1118-1138.

[Ish] H. Ishii, Perron's method for Hamilton-Jacobi equations, Duke Math. J. 55 (1987), 369-384. 
[KL] R. V. Kohn and R. Lipton, The effective viscosity of a mixture of two Stokes fluids, "Advances in Multiphase Flow and Related Problems." (ed. G.Papanicolaou) (1986), 123-135, SIAM.

[MS] V. G. Maz'ya and T. O. Shaponikova, "Theory of Multipliers in Spaces of Differentiable Functions," Pitman, Boston-London-Melbourne, 1985.

[Sol 1] V. A. Solonnikov, On a nonstationary motion of a finite isolated mass of selfgravitating fluid, Algebra and analysis 1 (1989), 207-246.

[Sol 2] V. A. Solonnikov, On the evolution of an isolated volume of viscous incompressible capillary fluid for large values of time, Vestnik Leningrad. Univ. Math. 15 (1987), 52-58. (English translation from Vestnik Leningrad. Univ. 20(3), 49-55 (1987))

[Sol 3] V. A. Solonnikov, Unsteady motion of a finite mass of fluid, bounded by a free surface, J. Soviet Math. 40 (1988), 672-686. (English translation from Zap. Nauchn. Sem. LOMI 152, 137-157 (1986))

[Sol 4] V. A. Solonnikov, On the transient motion of an isolated volume of viscous incompressible fluid, Math. USSR-Izv. 31 (1988), 381-405. (English translation from Izv. Akad. Nauk SSSR Ser. Mat. 51, 1065-1087 (1987))

[Sol 5] V. A. Solonnikov, Solvability of a problem on the motion of a viscous incompressible fluid bounded by a free surface, Math. USSR-Izv. 11 (1977), 1323-1358. (English translation from Izv. Akad. Nauk SSSR Ser. Mat. 41, 1388-1424 (1977))

[Sol 6] V. A. Solonnikov, Solvability of the problem of evolution of an isolated volume of viscous incompressible capillary fluid, J. Soviet Math. 32 (1986), 223-228. (English translation from Zap. Nauchn. Sem. LOMI 140, 179-186 (1984))

[Sy] G. Sylvester, Large-time existence of small viscous surface waves without surface tension, Comm. Partial Differential Equations 15 (1990), 823-903.

[Tana] N. Tanaka, Global existence of two phase non-homogeneous viscous incompressible fluid flow, preprint.

[Tani 1] A. Tani, On the evolution equations of the two-phase compressible viscous capillary fluids, in preparation. (Announcement in: Free boundary problems for general fluids. Kokyuroku. RIMS. Kyoto Univ. 698, 146-170 (1988))

[Tani 2] A. Tani, Multi-phase free boundary problem for the equation of motion of general 
fluids, Comm. Math. Univ. Carolinae 26 (1985), 201-208.

[Tani 3] A. Tani, Two-phase free boundary problem for compressible viscous fluid motion, J. Math. Kyoto Univ. 21 (1981), 839-859.

[Tri] H. Triebel, "Interpolation Theory, Function Spaces, Differential Operators," NorthHolland, Amsterdam-New York-Oxford, 1978.

[Ya] M. Yamazaki, A quasi-homogeneous version of paradifferential operators, I. Boundedness on spaces of Besov type, J. Fac. Sci. Univ. Tokyo Sect. IA, Math. 33 (1986), 131-174. 